\title{
Gestão da Qualidade: conhecimento do enfermeiro que trabalha em hemodiálise
}

\author{
Quality Management: knowledge of nurses working in hemodialysis \\ Gestión de la Calidad: conocimiento de enfermeras que trabajan en hemodiálisis
}

Recebido: 21/11/2021 | Revisado: 01/12/2021 | Aceito: 06/12/2021 | Publicado: 16/12/2021

\author{
Saturnina Alves da Silva Martins \\ ORCID: https://orcid.org/0000-0003-0231-2633 \\ Universidade Paulista, Brasil \\ E-mail: sasmartins@hotmail.com \\ Pedro Luiz Oliveira Costa Neto \\ ORCID: https://orcid.org/0000-0002-6987-2996 \\ Universidade Paulista, Brasil \\ E-mail: pedroluiz@plocn.com
}

\begin{abstract}
Resumo
$\mathrm{Na}$ área da saúde, a gestão da qualidade relaciona-se à administração do projeto de sistemas, políticas e processos que reduzem, e até extingui danos que podem ocorrer ao paciente, aprimorando o atendimento. O Enfermeiro é um profissional essencial no gerenciamento e assistência prestada ao paciente, sua satisfação e a qualidade geral do atendimento deve envolver elementos que combinam cuidados eficazes e resultados com excelência neste atendimento. Este estudo teve como objetivo analisar o conhecimento do enfermeiro que trabalha em hemodiálise sobre gestão da qualidade. Trata-se de uma pesquisa de campo, exploratória e descritiva, com abordagem qualitativa, através de estudo de casos múltiplos. Participaram da pesquisa enfermeiros que trabalham em quatro clínicas de hemodiálise localizadas na zona leste da cidade de São Paulo. A análise dos dados coletados foi realizada através das transcrições das entrevistas e análise do discurso do sujeito, os resultados foram apresentados em quadros de síntese geral dos comentários. Foi possível perceber que os enfermeiros descrevem a gestão da qualidade em hemodiálise como uma estratégia importante que auxilia a assistência, otimiza os processos de trabalho envolvendo os vários profissionais que atuam neste segmento. Porém o conhecimento sobre gestão da qualidade é mencionado de forma fragmentada e não uniforme até mesmo para os enfermeiros que trabalham na mesma clínica de hemodiálise.
\end{abstract}

Palavras-chave: Gestão da qualidade; Enfermeiro; Hemodiálise.

\begin{abstract}
In the healthcare area, quality management is related to project administration of systems, policies and processes that reduce and even extinguish the damage that can occur to the patient, improving care. Nurses are essential professionals in the management and care provided to patients, their satisfaction and the general quality of care must involve elements that combine effective care and results with excellence in this care. This study aimed to analyze the knowledge of nurses working in hemodialysis on quality management. It is a field research, exploratory and descriptive, with a qualitative approach, through the study of multiple cases. Nurses who work in four hemodialysis clinics located in the east side of the city of São Paulo participated in the research. The analysis of the collected data was carried out through the transcripts of the interviews and analysis of the subject's speech, the results were presented in general summary tables of the comments. It was possible to notice that nurses describe quality management in hemodialysis as an important strategy that helps care, optimizes work processes involving the various professionals who work in this segment. However, knowledge about quality management is mentioned in a fragmented and non-uniform way, even for nurses who work in the same hemodialysis clinic.
\end{abstract}

Keywords: Quality management; Nurse; Hemodialysis.

\section{Resumen}

En el área de la salud, la gestión de la calidad está relacionada con la administración de proyectos de sistemas, políticas y procesos que reducen e incluso extinguen el daño que le puede ocurrir al paciente, mejorando la atención. El enfermero es un profesional imprescindible en la gestión y atención que se brinda a los pacientes, su satisfacción y la calidad general de la atención deben involucrar elementos que combinen una atención y resultados efectivos con la excelencia en esta atención. Este estudio tuvo como objetivo analizar el conocimiento de los enfermeros que trabajan en hemodiálisis sobre la gestión de la calidad. Es una investigación de campo, exploratoria y descriptiva, con enfoque cualitativo, a través del estudio de múltiples casos. En la investigación participaron enfermeras que laboran en cuatro clínicas de hemodiálisis ubicadas en el lado este de la ciudad de São Paulo. El análisis de los datos recolectados se realizó a través de las transcripciones de las entrevistas y análisis del discurso del sujeto, los resultados se presentaron en tablas resumen generales de los comentarios. Se pudo constatar que los enfermeros describen la gestión de la calidad en hemodiálisis como una estrategia importante que ayuda al cuidado, optimiza los procesos de trabajo 
involucrando a los diversos profesionales que laboran en este segmento. Sin embargo, el conocimiento sobre la gestión de la calidad se menciona de forma fragmentada y no uniforme, incluso para los enfermeros que trabajan en la misma clínica de hemodiálisis.

Palabras clave: Gestión de la calidad; Enfermero; Hemodiálisis.

\section{Introdução}

A definição de qualidade está associada a questões que abrange o relacionamento ético entre os envolvidos na fabricação e comercialização de um produto ou prestação de um serviço (Mello, 2010). Observa-se que a qualidade está associada à capacidade que um produto ou serviço consiga servir aos propósitos dos consumidores, mostrando excelência (Silva et al., 2018).

A prática de Qualidade surgiu a partir da década de 50, e pesquisadores como Armand V. Feigenbaum, Joseph M. Juran e Winston Edwards Deming desenvolveram vários estudos sobre o tema. Feigenbaum afirma que a qualidade envolve o processo de fabricação dos produtos. Juran descreve a qualidade como adequação ao uso, idealizou a trilogia da qualidade envolvendo o planejamento, a melhoria e o controle da qualidade como sendo fundamentais. Para Deming a qualidade envolvia o sentimento de orgulho de um trabalho realizado com excelência (Carpinetti, 2012).

Ao longo dos tempos a concepção de qualidade foi se transformando e surgindo o conceito de qualidade total, visando não apenas atender as necessidades dos clientes, mas também satisfazer todos os departamentos de uma organização (Silva et al., 2018). A Qualidade Total tornou-se um modelo de gestão operacionalizado pelas organizações que tem como foco a qualidade. Esse modelo está fundamentado na participação de todos os integrantes da organização, que visam o sucesso em longo prazo, através da satisfação dos clientes internos e externos (Silva et al., 2016).

O conceito de qualidade na área da saúde surgiu nos anos de 1950, nos Estados Unidos, com a criação em 1951 pela Joint Commission on Accreditation of Healthcare, com a proposta que os hospitais norte-americanos deveriam alcançar e cumprir parâmetros de qualidade no atendimento prestado (Hervás, 2011).

Outra contribuição importante foi do médico Avedis Donabedian, que em 1966 sugeriu três princípios de qualidade assistencial. O primeiro se refere a estrutura, que envolvem os meios materiais, humanos e formativos, assim como o espaço físico onde o serviço é realizado. O segundo critério que Donabedian indica são os processos considerados uma série de atividades realizadas pelos profissionais durante a assistência ao paciente. E o terceiro são os resultados que indicam quais mudanças foram produzidas no estado de saúde do paciente e sua satisfação com relação a atenção recebida (Hervás, 2011).

A partir de 1990 surgiram no Brasil ações relacionadas à acreditação hospitalar. Essas ações tinham como premissa estimular o desenvolvimento de programas de qualidade (Carvalho et al., 2004). Os processos de gestão da qualidade nas organizações de saúde foram intensificados em 1995, com a implementação da Portaria GM/MS nº. 1.107, conforme citado no Manual Brasileiro de Acreditação Hospitalar (Ministério da Saúde, 2002).

O processo de acreditação é um método de conformidade, racionalização e ordenação das organizações prestadoras dos serviços de saúde e, principalmente, de educação permanente dos seus profissionais. É um processo voluntário, em que a organização de saúde passa por auditorias externas para avaliar o cumprimento de critérios e padrões pré-estabelecidos por uma organização independente e que, conforme os resultados, receberá ou não a acreditação (Organização Nacional de Acreditação, 2018). Este processo é sistêmico e avalia todo o funcionamento da organização de saúde. A vantagem de se introduzir a gestão baseada na acreditação se relaciona à melhoria no gerenciamento, na execução de técnicas voltadas à qualidade e na satisfação do cliente, bem como à maior capacidade de se obter vantagem competitiva (D'innocenzo, 2006).

No que se refere a Gestão da Qualidade e os serviços de Hemodiálise no Brasil, em agosto do ano de 2002 foi criado o Manual de Acreditação de Serviços de Nefrologia e Terapia Renal Substitutiva, elaborado através de oficinas técnicas e ações conjuntas da Organização Nacional de Acreditação, Sociedade Brasileira de Nefrologia, Agencia Nacional de Vigilância 
Sanitária e Câmara Técnica de Vigilância Sanitária do Conselho Nacional dos Secretários Estaduais de Saúde. A elaboração deste manual visa aprimorar e tornar continua a prática da gestão da qualidade em Serviços de Terapia Renal Substitutiva que se enquadram as Clínicas de Hemodiálise (Ministério da Saúde, 2003).

Entre as variadas áreas de atuação do enfermeiro, evidencia-se a especialidade da Nefrologia, em que o profissional atua em unidades de internação e clínicas de hemodiálise. O paciente que realiza hemodiálise passa por uma mudança significativa no seu cotidiano. Com isso, o enfermeiro que trabalha em hemodiálise deve primar por uma assistência que perceba o indivíduo de maneira holística (Barbosa \& Valadares, 2009).

A operacionalização da hemodiálise requer do enfermeiro a observação constante dos sinais e sintomas apresentados e referidos pelo paciente durante o procedimento, a monitoração do funcionamento da máquina de hemodiálise e materiais utilizados e competência para analisar e tomar decisões assertivas à resolução de ocorrências, beneficiando assim a segurança do paciente (Lima et al., 2010).

Segundo o último Censo da Sociedade Brasileira de Nefrologia publicado em 2020, no ano de 2018 no Brasil estavam cadastradas 786 clínicas de hemodiálise, englobando unidades satélites como clínicas intra-hospitalar. O Estado de São Paulo possui cadastradas 182 clínicas, destas, três possuem certificado de Acreditação.

A escolha deste estudo justifica-se como uma contribuição relevante, porque as questões que envolvem a gestão da qualidade na área da saúde despontam com um potencial para ampliar e habilitar novos processos relacionados à saúde e transformá-los em práticas utilizáveis no trabalho do enfermeiro que atua em hemodiálise.

Neste contexto o presente estudo pretende responder a seguinte questão de pesquisa: Qual o conhecimento do enfermeiro que trabalha em hemodiálise sobre o processo de gestão da qualidade?

Com a finalidade de responder ao questionamento proposto, esta pesquisa tem o objetivo de analisar o conhecimento do enfermeiro que trabalha em hemodiálise sobre gestão da qualidade.

\section{Metodologia}

Trata-se de uma pesquisa de campo, exploratória e descritiva, com abordagem qualitativa, através de estudo de casos múltiplos. Estudo de caso múltiplos, segue uma lógica da replicação e não de amostragem. A lógica da replicação é análoga aos experimentos múltiplos. Cada caso deve ser selecionado cuidadosamente para que possa predizer resultados similares ou possa produzir resultados contrastantes, mas para razões previsíveis (Yin, 2010).

A pesquisa de campo foi realizada em 4 clínicas de hemodiálise situadas na região leste da cidade de São Paulo. As clínicas foram categorizadas em HD1 a HD4, sendo que "HD" significa hemodiálise. Estas clínicas que fizeram parte do estudo não possuem selo de acreditação.

Os participantes da pesquisa constituíram-se de 7 enfermeiros, os enfermeiros foram categorizados em Enf.1 a Enf.7, sendo que "Enf." significa enfermeiro.

A coleta de dados foi realizada nos meses de fevereiro e março de 2021 por meio de entrevista com auxílio de um questionário semiestruturado com o auxílio de um gravador, tendo uma duração média de 45 minutos. Vale ressaltar que devido a pandemia do Coronavirus as entrevistas ocorreram na modalidade online utilizando a ferramenta de vídeo do aplicativo WhatsApp ${ }^{\circledR}$. As questões que foram norteadoras das entrevistas relacionavam a: conhecimento sobre gestão da qualidade, se o local onde trabalha utilizava alguma ferramenta de gestão da qualidade, se tinha conhecimento sobre processo de acreditação e sua importância, se havia algum formulário que o paciente respondesse sobre a satisfação do atendimento realizado no procedimento de hemodiálise.

Para realizar a articulação lógica que une os dados a questão de pesquisa e objetivo do estudo, foi utilizada a técnica de triangulação dos dados e a análise do discurso do sujeito, através das falas das entrevistas e a gravações realizadas que foram 
transcritas. A técnica de triangulação pode ser usada para validar os dados por meio da comparação entre fontes de dados distintas, examinando a evidência dos dados e usando-os para construir uma justificativa para os temas (Creswell, 2010). No que se refere a análise do discurso do sujeito é uma metodologia flexível, cujo objeto de estudo é a fala do entrevistado, em conjunto é realizado uma síntese geral de cada comentário realizado (Paulon et al., 2014). A análise do discurso do sujeito é um meio de expressar o significado implícito no discurso, por meio desta interpretação pode-se alcançar certos fenômenos e conceitos, as palavras ditas atuam como ponte entre o pesquisador e o entrevistado (Macedo et al., 2008).

Este estudo atende à Resolução 466/2012 do Conselho Nacional de Saúde, onde foram respeitados todos os aspectos éticos proposto. O projeto do estudo foi inscrito na Plataforma Brasil e direcionado ao Comitê de Ética e Pesquisa, com o Certificado de Apresentação de Apreciação Ética (CAAE): 30947019.2.0000.5512, número do parecer 4.072.867, aprovado em 05.06.2020, tendo como Instituição Proponente a Associação Unificada Paulista de Ensino Renovado Objetivo-Assupero.

\section{Resultados e Discussão}

Nesta sessão serão apresentados e discutidos os dados coletados, por meio das entrevistas realizadas. A discussão visa relacionar o que foi identificado na pesquisa de campo realizada com sete enfermeiros que atuam nas clinicas de hemodiálise HD1, HD2, HD3 e HD4, sobre o tema gestão da qualidade.

A Terapia Renal Substitutiva que inclui o procedimento de hemodiálise tem como foco a promoção e recuperação da saúde do paciente portador de Insuficiência Renal Cronica. O método de hemodiálise tem como objetivo substituir a função dos rins em depurar o sangue das escórias metabólicas e excesso de água e sais do organismo (Martins et al., 2014).

Além da organização de saúde ser estruturada em sua finalidade, política e filosofia como empresa, essa organização também tem por objetivo assegurar a manutenção da qualidade dos serviços prestados, garantindo o desenvolvimento dessa característica para atingir níveis de excelência cada vez maiores, conforme a necessidade do mercado (Balsanelli et al., 2008).

Os sujeitos da pesquisa foram sete enfermeiros que atuam nas clinicas de hemdiálise, compondo: 3 enfermeiros da HD1, 1 enfermeiro da HD2, 2 enfermeiros da HD3 e 1 enfermeiro da HD4. O processo de trabalho do enfermeiro abrange instrumentos, propósitos, métodos e produtos. Como integrantes da equipe de saúde, os enfermeiros têm prerrogativas especificas (Sanna, 2007). O enfermeiro atua tanto na assistência como na gerência do cuidado. Esta prestação de serviço visa a excelência da qualidade na atenção à saúde oferecida ao paciente, família e coletividade, com intervenção no processo saúdedoença (Carvalho \& Bachion, 2009).

A seguir, serão apontados em forma de Quadros de resultado total, a sintese dos comentários produzidos de formas semelhantes pelos enfermeiros, conforme distribuição das tematicas elencadas de maior relevancia para o estudo.

\subsection{Gestão da qualidade em hemodiálise}

Quadro 1. Comentários dos enfermeiros sobre gestão da qualidade em hemodiálise.

\begin{tabular}{|c|c|c|}
\hline \multicolumn{2}{|c|}{ Síntese dos Comentários dos Enfermeiros } \\
\hline HD3 & Enf.1 & Ferramenta para organizar e facilitar o trabalho. \\
\hline HD1 & Enf.2 & Gerência da qualidade do serviço prestado, e satisfação do paciente na \\
HD3 & Enf.4 & assistência prestada. \\
HD2 & Enf.6 & \\
\hline HD1 & Enf.3 & Estratégia de rever constantemente, todas as frentes do trabalho que se oferece \\
HD1 & Enf.5 & , devendo ter excelência, envolvendo parceiros , recursos humanos , \\
HD4 & Enf.7 & distribuidores, com padronização de rotinas. \\
\hline
\end{tabular}

Fonte: Autores (20210. 
Observa-se que os enfermeiros percebem a gestão da qualidade em hemodiálise como uma estratégia que envolvem além da prestação da assistencia com qualidade ao paciente, uma forma também de melhorar os processos de trabalho envolvendo os vários profissionais que atuam neste segmento.

A qualidade é um dos principios mais importantes da gestão. Manter as esferas da qualidade em cada estilo da organização, ajuda a atrair clientes fieis, mantem um fluxo de caixa regular e incita a concorrência (Bhasin, 2019).

Qualidade na saúde é fazer as coisas certas para as pessoas certas no momento certo, e fazê-las certo da primeira vez e sempre, também pode se referir à qualidade técnica do atendimento, aos aspectos não técnicos da prestação de serviços, como o tempo de espera dos pacientes e as atitudes dos funcionários, e a elementos programáticos, como políticas, infraestrutura, acesso e gerenciamento (Dodwad, 2013).

As clinicas de hemodiálise se empenham em viabilizar aos seus pacientes cuidados de saúde com qualidade adaptados às suas expectativas. A fim de alcançar os padrões de qualidade desejados e propiciar eficácia e confiabilidade, optam cada vez mais pela implantação de um sistema de gestão da qualidade (Kavalieratou et al., 2021).

\subsection{Adoção de Sistema de Gestão da Qualidade}

Quadro 2. Comentários dos enfermeiros sobre adoção de sistema de gestão da qualidade.

\begin{tabular}{|c|c|c|}
\hline \multicolumn{3}{|r|}{ Síntese dos Comentários dos Enfermeiros } \\
\hline HD3 & Enf.1 & É utilizado fluxograma. \\
\hline $\begin{array}{l}\text { HD1 } \\
\text { HD1 } \\
\text { HD3 } \\
\text { HD2 } \\
\text { HD4 }\end{array}$ & $\begin{array}{l}\text { Enf.2 } \\
\text { Enf.3 } \\
\text { Enf.4 } \\
\text { Enf.6 } \\
\text { Enf.7 }\end{array}$ & $\begin{array}{l}\text { Entrevistas com os pacientes sobre a satisfação do serviços prestado, } \\
\text { reciclagens com os funcionários, rotinas escritas disponíveis para consulta, } \\
\text { devolução de técnicas, avaliação de desempenho, reuniões multidisciplinar, } \\
\text { avaliação de relatórios de custos, controle dos resultados dos exames dos } \\
\text { pacientes e planos de ações para atender a demanda das necessidades dos } \\
\text { pacientes. }\end{array}$ \\
\hline HD1 & Enf.5 & Procedimento de operação padrão, manual de segurança do paciente. \\
\hline
\end{tabular}

Fonte: Autores (2021).

Foi possível perceber que os enfermeiros identificam os sistemas de gestão da qualidade implementados nas clinicas de hemodiálise em que trabalham de maneira especifica.

O segmento que envolve foco no cliente e gestão de relacionamento, foi relatado as entrevistas que são realizadas com os pacientes, planos de ações para atender a demanda das necessidades dos pacientes, controle dos resultados dos exames e o manual de segurança do paciente.

Em relação a abordagem de processos pode-se perceber as questões que envolvem o fluxograma, rotinas escritas disponíveis, avaliação de relatórios de custos.

No que se refere a melhoria continua e engajamento das pessoas, foi identificado a reciclagem com os funcionários, devolução de técnicas, avaliação de desemepnho e reuniões multidisciplinar.

Tomada de decisão com base em evidência pode-se indicar o controle dos resultados dos exames e os planos de ações para atender a demanda das necessidades dos pacientes.

O que chamou a atenção dos pesquisadores que as questões que envolvem liderança de uma maneira geral, não foi mencionado.

Dentro das organizações de saúde tendo a atuação da equipe de enfermagem na assistencia prestada pelo técnico e ou auxiliar de enfermagem é prerrogativa legal o enfermeiro liderar esta equipe. É o enfermeiro quem deve assumer a liderança do processo de trabalho cooperando nas diversas ações que a equipe realiza durante a assistência e a execução do cuidado (Rodrigues \& Cardoso, 2019). 
Um Sistema de Gestão da Qualidade é um conjunto de políticas, processos e procedimentos necessários para planejamento e execução na área do negócio principal de uma organização que pode impactor a capacidade de atender seus clientes internos e externos, a ISO 9001:2015 é um exemplo de Sistema de Gestão da Qualidade (Mourougan \& Sethuraman, 2017).

A ISO 9001:2015 auxilia as organizações a garantirem que seus clientes recebam de forma consistente alta qualidade nos produtos e serviços ofertados, com isso muitos beneficios, incluindo clientes e funcionários satisfeitos atraindo mais lucros para a organização (Mourougan \& Sethuraman, 2017).

Para atender as demandas das organizações de saúde em relação a implementação dos processos de gestão da qualidade no que se refere a ISO 9001:2015 algumas adaptações foram direcionadas para atendimento da demanda dos pacientes que acabam sendo clientes diferenciados por envolver um processo de saúde e doença (Tošić et al., 2018). A qualidade no fornecimento de serviços nas organizações de saúde é indicada como um elemento indispensável, buscando potencializar o bem estar e a satisfação das pessoas que procuram tratamento para sua doença (Lima \& Acuña, 2020).

\subsection{Realização de Procedimentos de Operação Padrão}

Quadro 3. Comentários dos enfermeiros sobre realização de Procedimentos de Operação Padrão.

\begin{tabular}{|l|l|l|}
\hline \multicolumn{2}{|c|}{ Síntese dos Comentários dos Enfermeiros } \\
\hline HD3 & $\begin{array}{l}\text { Enf.1 } \\
\text { Enf.4 }\end{array}$ & $\begin{array}{l}\text { Higiene das mãos, instalar e desinstalar o paciente na máquina de hemodiálise, } \\
\text { realização do curativo em cateteres, realização de punção de Fistula Arterio } \\
\text { Venosa, desperoxidação do capilar, técnica para preparo e administração de } \\
\text { medicamento. }\end{array}$ \\
\hline HD1 & $\begin{array}{l}\text { Enf.2 } \\
\text { Enf.3 } \\
\text { Enf.5 }\end{array}$ & $\begin{array}{l}\text { Higiene das mãos, regras de isolamento, admissão do paciente, lavagem de } \\
\text { capilar, limpeza das máquinas de hemodiálise, limpeza da ambulância. }\end{array}$ \\
\hline HD2 & Enf.6 & $\begin{array}{l}\text { Auxílio na inserção de cateter de duplo lumem para hemodiálise, coleta de } \\
\text { sangue antes da sessão de hemodiálise, preparo de capilar novo, coleta de } \\
\text { hemocultura, realização de curativo aberto e fechado. }\end{array}$ \\
\hline HD4 & Enf.7 & $\begin{array}{l}\text { Higienizar o local do procedimento de hemodiálise, pesagem dos pacientes, } \\
\text { punção da Fistula Arterio Venosa, conferência do capilar, controle dos } \\
\text { sinais vitais. }\end{array}$ \\
\hline
\end{tabular}

Fonte: Autores (2021).

Todos os enfermeiros relatam a institucionalização de Procedimento de Operação Padrão para várias atividades de assistencia prestada ao paciente em procedimento de hemodiálise.

A gestão da qualidade na área da saúde requer um processo de padronização. O Procedimento de Operação Padrão é uma representação sistemática, seguindo um passo a passo crítico e sequencial que deverá ser realizado pelo profissional de saúde, garantindo um resultado esperado da assistencia prestada (Almeida et.al, 2017).

Conforme é descrito na Resolução RDC no 154 de 15 de junho de 2004, estabelecendo o regulamento técnico para o funcionamento dos serviços de diálise, no item 5 Procedimento do Serviço de Diálise, todo o serviço deve estabelecer, por escrito, em conjunto com o responsavel do Programa de Controle e Prevenção de Infecção e de Eventos Adversos, uma rotina de funcionamento, assinada pelo responsável técnico e pelo enfermeiro responsável pelo serviço de hemodiálise, compatível com as exigencies técnicas previstas no regulamento (Ministério da Saúde, 2004). 


\subsection{Metodologias de Gestão da Qualidade}

Quadro 4. Comentários dos enfermeiros sobre o conhecimento de metodologias de gestão da qualidade.

\begin{tabular}{|l|l|l|}
\hline \multicolumn{2}{|c|}{ Síntese dos Comentários dos Enfermeiros } \\
\hline HD3 & Enf.1 & Fluxograma \\
\hline HD1 & Enf.2 & Protocolos de atendimento \\
\hline HD1 & Enf.3 & $\begin{array}{l}\text { Avaliação entre o grupo de trabalho, para envolvimento e engajamento dos } \\
\text { funcionários. Foi ressaltado que as atividades são realizadas de forma empírica, } \\
\text { faltando empregar metodologias especificas. }\end{array}$ \\
\hline HD3 & Enf.4 & Relata que já ouviu falar, mas não soube citar nada a respeito. \\
\hline HD1 & Enf.5 & Manuais e Procedimento de Operação Padrão \\
\hline HD2 & Enf.6 & $\begin{array}{l}\text { Gestão de mudança, gestão de desenvolvimento, gestão de qualidade no } \\
\text { tratamento }\end{array}$ \\
\hline HD4 & Enf.7 & Gráficos e Controles \\
\hline
\end{tabular}

Fonte: Autores (2021).

Evidencia-se que em relação as metodologias de gestão da qualidade os enfermeiros não relatam de maneira clara sobre o conhecimento das metodologias, apesar que nos Quadros 1, 2 e 3 foi percebido pelos pesquisadores que é utilizado estas metodologias nas clinicas de hemodiálise, conforme depoimentos nas entrevistas realizadas.

As metodologias de gestão da qualidade envolve quatro principios:

$\checkmark \quad$ Planejamento da qualidade: que é conceber um plano de gerenciamento da qualidade que descreve os processos e métricas que devem ser usados, garantia da qualidade;

$\checkmark$ Garantia da qualidade: envolve assegurar, validar e exibir para a organização que as lideranças envolvidas e toda equipe de trabalho possui habilidades, conhecimento e atitudes para atingir o resultado desejado;

$\checkmark$ Controle da qualidade: está relacionado a inspeção, testes e medições das atividades realizadas e entrega do resultado final;

$\checkmark$ Melhoria Continua: envolve a supervisão e avaliação do planejamento, garantia e controle da qualidade.

Supervisionar e manter estes principios alinhados são necessários para manter ou atingir um determinado nível de excelência na organização (Garvey, 2019). 


\subsection{Selo de Acreditação para Clínica de Hemodiálise}

Quadro 5. Comentários dos enfermeiros sobre a importância do selo de Acreditação para clínica de hemodiálise.

\begin{tabular}{|l|l|l|}
\hline \multicolumn{3}{|c|}{ Síntese dos Comentários dos Enfermeiros } \\
\hline HD1 & $\begin{array}{l}\text { Enf.2 } \\
\text { Enf.3 } \\
\text { Enf.5 }\end{array}$ & $\begin{array}{l}\text { Atender os pacientes com maior segurança. Facilidade na realização dos } \\
\text { procedimentos, satisfação e excelência de atendimento. Há dificuldade na } \\
\text { contratação do serviço das organizações de acreditação. Seria vista como uma } \\
\text { clínica que presta serviço de qualidade, atualmente a clínica realiza } \\
\text { procedimentos considerados com padrão de qualidade, porém não tem o selo } \\
\text { acreditação. }\end{array}$ \\
\hline HD2 & $\begin{array}{l}\text { Enf.6 } \\
\text { Enf.1 } \\
\text { Enf.4 }\end{array}$ & \begin{tabular}{l} 
Projetar um serviço com qualidade e melhorar a qualidade do serviço prestado. \\
Manter a padronização dos processos e alcançar a satisfação dos pacientes \\
\hline HD4
\end{tabular} Enf.7 \\
\hline
\end{tabular}

Fonte: Autores (2021).

Os enfermeiros percebem a importancia da conquista de um selo de Acreditação para a clinica de hemodiálise, envolvendo facilidade na realização dos procedimentos, direcionando um cuidado seguro ao paciente, maior projeção da organização e satisfação dos pacientes em realizar o procedimento para melhorar a qualidade de vida em uma clinica com selo de Acreditação. Vale ressaltar que o Enf. 3 relata na entrevista a dificuldade da contratação de organizações acreditadoras devido ao custo elevado na contratação do serviço.

A inscrição no processo de avaliação e contratação da instituição acreditadora segue algumas fases:

a) A organização de saúde manifesta interesse para ser avaliada junto a uma instituição acreditadora;

b) A instituição acreditadora coleta as informações necessárias sobre a organização de saúde;

c) A instituição acreditadora encaminha proposta a organização de saúde.

Após analise da proposta e envio da documentação legal (alvará de funcionamento, licença sanitaria entre outros) referente a organização de saúde, a instituição acreditadora encaminha a Organização Nacional de Acreditação cópia do contrato firmado. É necessário a organização de saúde recolher uma taxa de $10 \%$ do valor do contrato firmado para realização da inscrição junto a Organização Nacional de Acreditação. Os custos totais da visita para a Acreditação é integralmente pago pela organização de saúde a instituição acreditadora (Ministério da Saúde, 2002).

O empenho para ter uma organização de saúde acreditada envolve à oportunidade de crescimento pessoal e profissional, estabilidade da organização com critério de valor diferenciado na prestação dos serviços, estimulo a melhoria continua dos processos e da assistencia ao paciente, confiança da sociedade e orgulho em prestar assistencia em uma organização acreditada (Manzo et al., 2013).

Especificamente para os serviços de hemodiálise em agosto de 2002, foi criado o Manual de Acreditação de Serviços de Nefrologia e Terapia Renal Substitutiva, elaborado através de oficinas técnicas e ações conjuntas da Organização Nacional de Acreditação, Sociedade Brasileira de Nefrologia, Agencia Nacional de Vigilância Sanitária e Câmara Técnica de Vigilância Sanitária do Conselho Nacional dos Secretários Estaduais de Saúde. A aprovação final ocorreu em 12 de novembro de 2003 (Ministério da Saúde, 2003).

Segundo o mapa da Organização Nacional de Acreditação, no Brasil há 16 clinicas de hemodiálise com algum nível de selo de Acreditação (Organização Nacional de Acreditação, 2020). No ano de 2018 o Brasil estava com 786 clinicas de hemodiálise cadastradas e em funcionamento, citado no ultimo censo da Sociedade Brasileira de Nefrologia. 


\section{Considerações Finais}

Este estudo fornece dados qualitativos sobre o conhecimento do enfermeiro que trabalha em hemodiálise, sobre o processo de gestão da qualidade.

Através das entrevistas realizadas e a análise do discurso, foi possível perceber que os enfermeiros relatam vários processos e ferramentas do sistema de gestão da qualidade, porém este conhecimento é fragmentado e não é uniforme até mesmo aos enfermeiros que trabalham na mesma clínica de hemodiálise.

De acordo com a Lei do Exercício Profissional de Enfermagem ño.7.498 de 1986, o enfermeiro exerce todas as atividades de enfermagem, sendo privativo às funções de: direção do órgão de enfermagem integrante da estrutura básica da instituição de saúde, pública e privada, organização e direção dos serviços de enfermagem, planejamento, organização, coordenação, execução e avaliação dos serviços da assistência de enfermagem.

Denota-se que o Enfermeiro tem um papel importante no processo da gestão da qualidade desde o diagnóstico situacional, planejamento e implementação das ações e avaliação contínua do processo. E para que isso tenha êxito é necessário que o Enfermeiro se aproprie do conhecimento técnico e científico sobre o que é qualidade e os processos de gestão da qualidade.

Como limitação do estudo aponta-se o quantitativo da amostra dos sujeitos de pesquisa e as dificuldades encontradas em realizar as entrevistas devido as restrições com o distanciamento social em decorrência da pandemia do Coronavirus.

Para pesquisas futuras sugere-se um aumento da amostra dos sujeitos de pesquisa, podendo ser utilizado uma abordagem quantitativa e análise descritiva.

\section{Agradecimentos}

Este estudo foi parcialmente financiado pela Coordenação de Aperfeiçoamento de Pessoal de Nível Superior - Brasil (CAPES) - Código Financeiro 001

\section{Referências}

Almeida, L. P. Cortez, E. A. Valente, G. S. C Rego, S. M. Ferreira, A. F. \& Fernandes, A. C. M. (2017). A não utilização dos procedimentos operacionais padrão por profissionais de saúde em um centro de diálise. Revista Enfermagem Atual Edição Especial.

Balsanelli, I. C. Cunha, K. O. Feldman, L. B. \& Ruthes, R. M. (2008). Competências gerenciais: desafio para o enfermeiro. Martinari.

Bhasin, H. (2019). What is the Importance of Quality Management for an Organization? https://www.marketing91.com/importance-of-quality-management/

Carpinetti, L. C. R. (2012). Gestão da Qualidade: conceitos e técnicas. Atlas.

Carvalho, C. O. M. Sardenberg, C. Matos, A. C. Cendoroglo Neto \& M. Santos, B. F. C. (2004). Qualidade em Saúde: Conceitos, Desafios e Perspectivas. J Bras Nefrol Volume XXVI - no 4.

Carvalho, E. C., \& Bachion, M. M. (2009). Processo de enfermagem e sistematização da assistência de enfermagem - intenção de uso por profissionais de enfermagem. Rev. Eletr. Enf. 11(3):466. http://www.fen.ufg.br/revista/v11/n3/v11n3a01.htm

Creswell, J. W. (2010). Projeto de Pesquisa: métodos qualitativo, quantitativo e misto. Tradução LOPES, M. (3a ed.), Artmed.

D'innocenzo, M. (2006). Indicadores, auditorias, certificações: ferramentas de qualidade para gestão em saúde. Martinari.

Dodwad S. S. (2013). Quality management in healthcare. Indian journal of public health, 57(3), 138-143. https://doi.org/10.4103/0019-557X.119814.

Garvey, J. (2019). Total quality management methods to drive têm performance. https://www.peoplegoal.com/blog/quality-management-techniques-to-driveteam-performance

Hervás, M. J. W. (2011). Sistema de Gestão da Qualidade. In: Luongo, J. et al. Gestão de Qualidade em Saúde. Rideel.

Kavalieratou, A. Garofyllou, G. Evangelidou, E. \& Mantzanas, M. (2021). Quality Management System in a Haemodialysis Unit and Patient Satisfaction. SciMedicine Journal,

Lei no.7.498. (1986). http://www.planalto.gov.br/ccivil_03/leis/17498.htm 
Lima, A. F. C. Fuzii, S. M. O. Pinho, N. A. Melo, A. C. T. \& Hashimoto, T. H. F. (2010) Processo de Enfermagem na prática de hemodiálise: a experiência das enfermeiras de um Hospital Universitário. Revista Referencia II Serie (12), 39-45.

Lima, J. A., \& Acuña, G. S. (2020). Evaluation of the quality of cérvices through the SERVQUAL model: a case study in a medical clinic of Pau dos Ferros City, Rio Grande do Norte State, Brazil. Research, Society and Development, 9(8), e960986427. https://doi.org/10.33448/rsd-v9i8.6427

Macedo, L. C. Larocca, L. M. Chaves, M. M. N. \& Mazza, V. A. (2008). Analysis of speech: a reflection on health research. Interface - Comunicação, Saúde, Educação, Botucatu, 12(26), $649-657$.

Manzo, B. F. Brito, M. J. M. \& Alves, M. (2013). Influência da comunicação no processo de acreditação hospitalar. Revista Brasileira de Enfermagem. Brasília, 66(1): 46-51.

Martins, S. A. S. Vechhi, R. A. \& Lima, W. A. (2014). O paciente em tratamento dialítico. In: Souza, A.B.G.; Chaves, L.D.; Silva, M.C. Enfermagem em Clínica Médica e Cirúrgica, teoria e prática. Martinari.

Mello, C. H. P. (2010). Gestão da Qualidade. Academia Pearson.

Ministério da Saúde. (2002). Manual Brasileiro de Acreditação Hospitalar. Série A. Normas e Manuais Técnicos; n. 117 (3a ed.), Revista e Atualizada Brasília - DF.

Ministério da Saúde. (2003). Agência de Vigilância Sanitária. Manual de Acreditação das Organizações Prestadoras de Serviços de Nefrologia e Terapia Renal Substitutiva. $1^{a}$ Edição. ONA. http://bvsms.saude.gov.br/bvs/publicacoes/MR_completo.pdf Acesso em 08.02.2020.

Ministério da Saúde. (2004). Agência Nacional de Vigilância Sanitária. Resolução - RDC nº 154 Regulamento Técnico para o funcionamento dos Serviços de Diálise. https://bvsms.saude.gov.br/bvs/saudelegis/anvisa/2004/rdc0154_15_06_2004_rep.html

Mourougan, S. \& Sethuraman, K. (2017). Understanding and Implementing Quality Management System. IOSR Journal of Business and Management (IOSRJBM) 19(5), 41-51 www.iosrjournals.org

Organização Nacional de Acreditação. (2018). Manual das Organizações Prestadoras de Serviços de Saúde. Coleção Manual Brasileiro de Acreditação.

Organização Nacional de Acreditação. (2020). https://www.ona.org.br/mapa-de-acreditacoes

Paulon, A. Nascimento, J. V. \& Laruccia, M. M. (2014). Análise do Discurso: Fundamentos Teórico-Metodológicos. Revista diálogos interdisciplinares, 3(1).

Rodrigues, F. C. P. \& Cardoso, C. T. C. (2019). A importancia da liderança do enfermeiro na configuração do processo de trabalho da enfermagem. Revista Interdisciplinar em Ciências da Saúde e Biológicas, 3(2)13-23.

Sanna, M. C. (2007). Os processos de trabalho em Enfermagem. Rev Bras Enferm. 60(2):221-4.

Silva, A. S. B. Bernardo, A. C. F. Ribeiro, D. Marier, J. B. A. \& Belline, Y. P. (2016). Qualidade Total: os princípios de Deming em uma multinacional. XXXVI Encontro Nacional de Engenharia de Produção Contribuições da Engenharia de Produção para Melhores Práticas de Gestão e Modernização do Brasil. João Pessoal - PB Brasil, de 03 a 06 de outubro.

Silva, V. L. Ferreira, D. V. Pereira, R. T. Silva, T. B. Alencar, B. M. Silva, M. M. Sousa, J. L. S. Ribeiro, J. V. S. \& Ibiapina, J. O. O. (2018). Gestão da Qualidade: Conceitos e Perspectivas em Segurança nos Serviços de Saúde Pública. Semana Acadêmica Revista Científica.

Sociedade Brasileira de Nefrologia. (2020). Censo Brasileiro de Diálise: análise de dados da década 2009-2018. Jornal Brasileiro de Nefrologia. 42 (2):191200 .

Tošić, B. Ruso, J. \& Filipović, J. (2018). Quality Management in health care: concepts, principles and standards. $3^{\text {rd }}$ International Conference on Quality of Life. http://cqm.rs/2018/cd1/pdf/papers/focus_1/33.pdf

Yin, R. K. (2010). Estudo de Caso-Planejamento e Métodos. (4a ed.), Bookman. 